\title{
Evaluación de la Sustitución de Grasa Animal por Grasa Vegetal Insaturada en la Elaboración de un Embutido de Carne de Búfalo (Bubalus bubalis)
}

\author{
Javier F. Rey Rodríguez y Lucila Gualdron \\ Programa de Ingeniería de Alimentos, Universidad de La Salle, Carrera 2 No 10-70 piso 7 bloque \\ D, Bogotá-Colombia (e-mail: jrey@unisalle.edu.co)
}

Recibido Jun. 16, 2010; Aceptado Ago. 18, 2010; Versión Final recibida Ago. 23, 2010

\begin{abstract}
Resumen
Se evaluó la sustitución de grasa animal por grasa vegetal en un producto escaldado utilizando la carne de búfalo en un salchichón seleccionado por su aceptabilidad en Colombia. Las formulaciones experimentales fueron preparadas por triplicado utilizando aceite de soya, canola y girasol en $5 \%, 10 \%$ y $15 \%$, estas fueron comparadas frente a un patrón, en el cual se utilizo carnes de vacuno y grasa animal. La humedad, proteína, grasa y textura (Warner-Bratzler) fueron determinadas por duplicado. La aceptabilidad fue evaluada con panel sensorial no entrenado. Los datos experimentales fueron evaluados mediante estadística descriptiva con análisis varianza multivariado y univariado, y establecieron que no hubo diferencias significativas entre las diferentes formulaciones con soya y girasol. El análisis sensorial definió como mejor producto el salchichón resultante del aceite de soya al $10 \%$.
\end{abstract}

\section{Evaluation of the Substitution of Animal Fat by Vegetable fat in the Manufacture of a Heat Processed Buffalo (Bubalus bubalis) Meat Sausage}

\begin{abstract}
The substitution of animal fat by vegetable fat in a heat processed meat sausage, made of buffalo meat, was evaluated. The meat sausage developed was salchichón, a product widely accepted by consumers in Colombia. Experimental formulations of meat sausage were prepared in triplicate with soybean, sunflower and canola oils at 5, 10 and 15\% concentrations. These formulations were compared with a standard formulation of meat sausage with animal fat. Moisture, protein, fat and texture (Warner-Bratzler) were determined in duplicate. Acceptability was evaluated by an untrained sensory panel. The experimental results were subjected to univariate and multivariate analyses of variance. No significant differences were found between the formulations with soybean and sunflower oils. The formulation with $10 \%$ soybean oil was defined as the best product.
\end{abstract}

Keywords: salchichón, soybean, sunflower, texture, sensory analysis. 


\section{INTRODUCCIÓN}

La carne de búfalo se caracteriza por bajos contenidos de grasa, colesterol y calorías en comparación con el pollo y la gran mayoría de los pescados y con más proteínas que los anteriores, contiene omega 3 y solo el 1,8 \% de sus cortes contiene colesterol (Escalante, 2007). La carne de búfalo contiene $70 \%$ y $90 \%$ menos grasa que la carne de vaca; en cambio contiene más proteína, hierro, aminoácidos de Omega 3. El interés en la producción del búfalo, es cada vez mayor, debido a la alta calidad de sus productos y su adaptabilidad a las condiciones ambientales (Lourenço, 2002).

En Argentina se han desarrollando investigaciones sobre la carne de búfalo como alimento saludable, las cuales han demostrado los beneficios en salud que se derivan del consumo de su carne, gracias a sus altos contenidos en proteína y sus contenidos bajos en ácidos grasos saturados y colesterol (Cedres et al. 2002). En Belén de Para, Brasil la carne bufalina se constituyo en una alternativa de alimentación para la población (Antunes et al. 2002).

En Colombia se han realizado estudios sobre análisis sensorial de la carne bufalina, y se ha concluido que los cortes de primera calidad de la carne bufalina, pueden ser comercializados ya que poseen, una buena aceptación, debido a sus propiedades organolépticas y a su alta aceptación sensorial (Hurtado-Lugo et al. 2004).

Actualmente la industria de los alimentos en Estados Unidos, esta incorporando estos ácidos grasos esenciales en los procesos industriales de freído en snacks, en leches fortificadas, en suplementos nutricionales, en elaboración de productos de panadería entre otras industrias (Duxbury 2005). En España se han desarrollado productos de esta índole, se han procesado cárnicos con menor contenido calórico; mejorando la calidad de aminoácidos, altos en proteínas; enriquecidos con fibra; incorporando prebiótico y probióticos en su formulación (Serrano y Cofrandes 2005).

El estudio realizado tuvo como fin principal la elaboración de salchichón bajo en ácidos grasos saturados aprovechando los cortes secundarios de la carne de búfalo. El desarrollo experimental se enfoca en tres etapas, la primera comprende el análisis de la carne de búfalo: capacidad de retención de agua, $\mathrm{pH}$, humedad, cenizas; la segunda etapa, estudia las formulaciones para elaborar el producto y una tercera, el seguimiento analítico de los productos para comprobar su calidad, enmarcada en las normas legales de la legislación colombiana. Una vez los productos fueron estandarizados se realizó un panel sensorial que junto a las pruebas analíticas determinaron las mejores formulaciones, comprobación a través de métodos estadísticos.

\section{MATERIALES Y MÉTODOS}

La presente investigación se realizó en la Planta Piloto de Carnes, laboratorio de Analisis de Alimentos y el laboratorio de Ingeniería Ambiental y Sanitaria de la Universidad De La Salle.

Se trabajó con la carne de segunda del búfalo (Bubalus bubalis), proporcionada por la empresa BOS INDICUS, para la sustitución de grasa se utilizaron los aceites de Soya, Girasol y Canola producidos por la empresa ALIANZA TEAM. La carne fue caracterizada mediante análisis físicoquímicos de proteína, grasa, humedad, cenizas y $\mathrm{pH}$ (protocolos $\mathrm{AOAC}$ ). Se aplicaron pruebas para establecer su capacidad de retención de agua (CRA) y su capacidad emulsionante (CE), siguiendo los procedimientos de Guerrero (1998) y finalmente se aplicaron pruebas de textura con cizalla Warner-Bratzler (Bourne, 2002 y Roudot, 2004), y utilizando un Chatillon DFS-100 (Loyds, Inglaterra).

Para definir la mejor formulación, se realizaron ensayos preliminares con los aceites seleccionados en diferentes porcentajes de grasa sustitutiva y así encontrar la composición definitiva. Se definieron las temperaturas máximas de estabilidad de los aceites vegetales en los procesos de emulsificación y escaldado siguiendo la formulación descrita por Ranken 2003, Tabla 1 , para emulsiones cárnicas. 
Tabla 1: Formulación para emulsiones cárnicas

\begin{tabular}{cc}
\hline Componentes & $\%$ \\
\hline Aceite & 20 \\
Carne & 59,8 \\
Hielo & 12 \\
Harina & 4 \\
Proteína de soya & 1 \\
Sal & 1,5 \\
Sabor & 1,2 \\
Estabilizante & 0.5 \\
\hline
\end{tabular}

Fuente: Ranken, 2003

Cada ensayo con los tres diferentes aceites fue sometido a los procesos de emulsificación de acuerdo con Lawrie (2006) y a escaldado siguiendo condiciones de proceso descritas por Ramírez (2006) y Lawrie (2006). Para el embutido se utilizó tripa en fibrosa transparente.

Estandarización del producto: Realizados los ensayos preliminares, se definieron las cantidades de aceite a incorporar (5\%, 10\% y 15\%) con tres repeticiones por cada formulación y las condiciones a las cuales debían ser utilizados los aceites en los diferentes procesos. A cada uno de los productos elaborados se les realizaron todos los análisis físico-químicos, sensoriales y de textura para definir mejor nivel de grasa en el producto, avalado con estudios estadísticos.

Análisis estadístico: Toda la información obtenida de los tratamientos fue analizada aplicando un análisis de varianza multivariado (MANOVA), buscando identificar si los diferentes niveles de aceite usados en la elaboración del salchichón introducen variaciones significativas en las características de los mismos. Para este análisis se aplico la matriz descrita en la tabla 2.

Tabla 2: Matriz del modelo estadístico utilizado para el producto salchichón.

\begin{tabular}{|c|c|c|c|c|c|c|c|c|}
\hline \multicolumn{2}{|c|}{ soya 5\% } & \multicolumn{3}{c|}{ soya 10\% } & \multicolumn{3}{c|}{ soya 15\% } \\
\hline \multicolumn{3}{|c|}{$0,5 \%$ est } & \multicolumn{3}{c|}{$1 \%$ est } & \multicolumn{3}{c|}{$1,5 \%$ est } \\
\hline rep1 & rep2 & rep3 & rep1 & rep2 & rep3 & rep1 & rep2 & rep3 \\
\hline \multicolumn{3}{|c|}{ girasol 5\% } & \multicolumn{3}{c|}{ girasol 10\% } & \multicolumn{3}{c|}{ girasol 15\% } \\
\hline \multicolumn{3}{|c|}{$0,5 \%$ est } & \multicolumn{3}{c|}{$1 \%$ est } & \multicolumn{3}{c|}{$1,5 \%$ est } \\
\hline rep1 & rep2 & rep3 & rep1 & rep2 & rep3 & rep1 & rep2 & rep3 \\
\hline
\end{tabular}

Para el análisis de esta información se procedió de la siguiente manera:

1. Análisis descriptivo de las características observadas en el producto cárnico. El objetivo fue visualizar el comportamiento de las características medidas en los diferentes niveles de aceite.

2. Análisis de varianza multivariado (MANOVA), para evaluar el efecto de las características del producto, donde la variable independiente es el nivel de grasa trans.

3. Análisis univariado de las características del producto, considerando una respuesta única, donde la variable independiente es el nivel de grasa trans y la dependiente es cada una de las mediciones (humedad, proteína, grasa y textura).

4. Elaboración de una única respuesta combinando las características del producto en una sola, considerando un peso para cada característica, donde este depende de la importancia de la característica en el producto.

\section{RESULTADOS Y DISCUSIÓN}

Caracterización de la materia prima: los análisis realizados a la carne de Búfalo se describen en la tabla 3 y se comparan con los obtenidos en con corte de segunda de bovino. 
Tabla 3: Composición química de la carne de segunda de búfalo y la de vacuno (100g de alimento)

\begin{tabular}{cccccc}
\hline Prueba & Proteína & Grasa total & Humedad & Cenizas & pH \\
\hline Búfalo & $24.7 \pm 0.8$ & $0.52 \pm 0.5$ & $74.3 \% \pm 1.7$ & $1.15 \% \pm 0.7$ & 5.8 \\
Vacuno & $20,43 \pm 1.0$ & $7,5 \pm 0.9$ & $70,5 \pm 1.2$ & $1,15 \pm 0.5$ & 6.0 \\
\hline
\end{tabular}

Se comprobó que la carne de búfalo posee mayor cantidad de proteína en comparación con la de bovino y predomina el bajo nivel de grasa, este resultado se debe a la baja concentración de grasa intramuscular que poseen los búfalos en su composición del músculo y a los alto niveles de hemoglobina que este animal tiene en su estructura muscular, estos resultados hacen importante el valor nutricional que tiene esta carne para el ser humano y las posibilidades que tiene para ser explotada como alimento o materia prima para transformación industrial.

Se analizó la capacidad emulsionante (CE) de la carne de búfalo y se encontró que es mayor a la de vacuno, ésta posee una CE de 1,061g aceite/g de proteína y la de búfalo ostenta una valor de $1,115 \mathrm{~g}$ de aceite/g de proteína, además tiene una capacidad de retención de agua (CRA) por goteo de $2,98 \%$ y por cocción de $53,5 \%$ esto se debe a la mayor proporción de hemoglobina en su músculo que hace que la carne se vuelva más funcional al momento de retener agua y grasa.

También se observa que la capacidad de retención de agua por goteo da unos resultados bajos que contrastan con la alta pérdida en el momento de la cocción, resultado que lleva a concluir que la adición de un estabilizante de la emulsión cárnica es necesaria para que las pérdidas por cocción en el escaldado y cocido de la carne no lleguen a superar el $2 \%$.

Por último, se analizó la terneza de la carne de búfalo con respecto a la de vacuno, mediante análisis de textura a través del método Warner-Bratzler encontrando que la primera necesita una fuerza de cizallamiento de 38,93 +/- 2,3 $\mathrm{N}$ mientras que la de vacuno necesita $30,91+/-1,8 \mathrm{~N}$ y esto significa que la carne de vacuno es de mayor terneza que la de búfalo y esto se debe a la mayor cantidad de fibra muscular que posee la carne de búfalo frente a la de bovino.

Ensayos preliminares: En la elaboración del producto se realizó siguiendo la formulación descrita en la Tabla 2, se trabajó con una base de cálculo de $2000 \mathrm{~g}$ de alimentación en la etapa de emulsificación, observándose una rotura de la emulsión a los 5 minutos de iniciado el proceso, subiendo la temperatura hasta $18^{\circ} \mathrm{C}$, con las tres muestras de aceite. Fue necesario subir la cantidad de estabilizante a $2 \%$, generando los mismos resultados de rotura de la emulsión en las mismas características, llegando a la conclusión de bajar el nivel de aceite en la formulación a $15 \%$ para cada uno de los tratamientos con soya, girasol, y canola, generando una nueva formulación (Tabla 4).

Tabla 4: Formulación para emulsión cárnica modificada.

\begin{tabular}{cc}
\hline Ingrediente & $\%$ \\
\hline Aceite & 15 \\
Carne & 64,8 \\
Hielo & 12 \\
Harina & 4 \\
Proteína de soya & 1 \\
Sal & 1,5 \\
Sabor & 1,2 \\
Estabilizante & 0.5 \\
\hline
\end{tabular}

Proceso de emulsificación: La formulación anteriormente descrita se puso a prueba en el proceso de emulsificación generando los resultados de temperaturas y tiempos de estabilidad de la emulsión como se aprecia en la Tabla 5, este proceso se realizó por triplicado.

Se concluyó que el máximo porcentaje de aceite a usar en la formulación es del 15\%. En la Figura 1 se puede observar el porcentaje promedio del tiempo y la temperatura para cada tipo de aceite, 
ilustrando así la variación presentada en el promedio de un tipo a otro. Se evidencia una variación alta cuando se cambia del aceite de soya al de canola, esto para tanto para el tiempo como para la temperatura, mientras que cuando para a girasol el comportamiento vuelve a ser similar al de soya.

Tabla 5: Tiempos y temperaturas de máxima estabilidad de los aceites en el proceso de emulsificación.

\begin{tabular}{cccc|ccc|ccc}
\hline Aceite & \multicolumn{3}{c|}{ Soya } & \multicolumn{3}{c|}{ Canola } & \multicolumn{3}{c}{ Girasol } \\
\hline Tiempo $(\mathrm{min})$ & 14.8 & 15.1 & 15.4 & 5.1 & 4.6 & 5.0 & 15.2 & 14.7 & 14.9 \\
Temperatura $\left({ }^{\circ} \mathrm{C}\right)$ & 10 & 12 & 12 & 8 & 5 & 7 & 10 & 10 & 11 \\
\hline
\end{tabular}

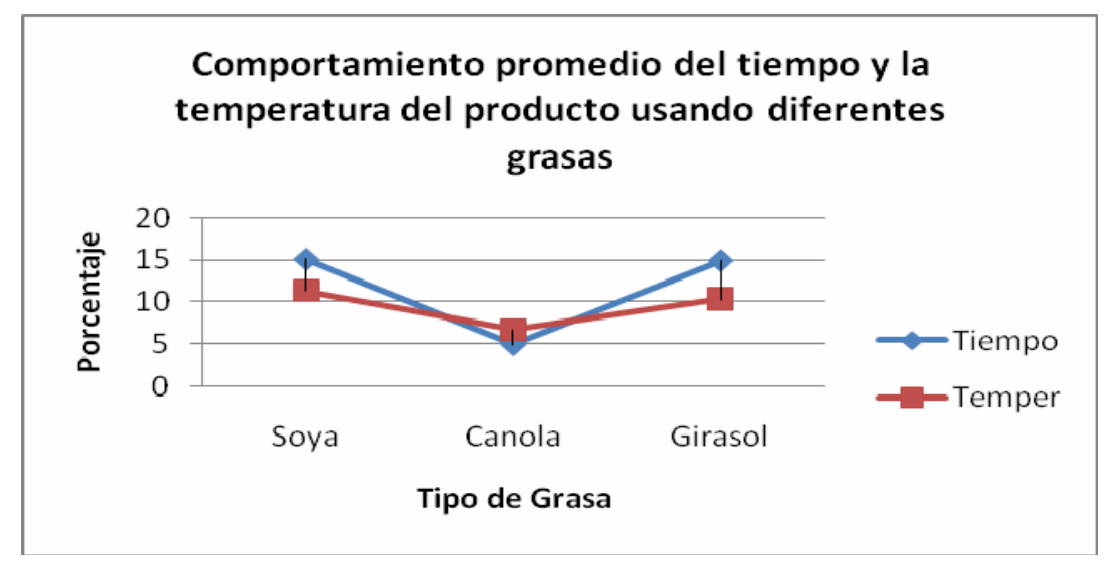

Fig. 1: Comportamiento promedio del tiempo y la temperatura en tres grasa.

En la Tabla 6 se encuentra el resultado obtenido con el MANOVA, el cual contiene el valor de la estadística $\mathrm{F}$, los grados de libertad usados para calcular el valor crítico y el $\mathrm{p}$-valor. El valor de la $\mathrm{F}$ permite establecer el efecto de las variables sobre las respuestas de interés (tiempo y temperatura).

Valores de P-valor menores al 0.05 indican que el tipo de aceite tiene un efecto significativamente diferente sobre las características consideradas en el producto, lo que significa que con diferentes tipos de grasa se obtendrán diferentes medidas de las características observadas. Para el MANOVA se consideraron tres estadísticas diferentes, las cuales aparecen en la primera columna de la Tabla 8: lambda de Wilks, traza de Hotelling-Lawley y raíz de Roy, con las tres se llega a la conclusión que el tipo de aceite no tiene efecto significativo sobre las características.

Tabla 6: Análisis de Varianza (MANOVA) para evaluar el efecto sobre el tiempo y la temperatura del producto

\begin{tabular}{ccccc}
\hline \multirow{2}{*}{ Estadística } & \multirow{2}{*}{ Valor de F } & \multicolumn{2}{c}{ Grados de libertad } & \multirow{2}{*}{ P-valor } \\
\cline { 3 - 4 } & & Numerador & Denominador & \\
\hline Lambda de Wilks & 76,22 & 4 & 10 & $<, 0001$ \\
Traza de Hotelling-Lawley & 1023,91 & 4 & 5 & $<, 0001$ \\
Raíz de Roy & 2502,34 & 2 & 6 & $<, 0001$ \\
\hline
\end{tabular}

Con respecto al aceite de canola se tiene una gran variación en el resultado con respecto a los de soya y girasol, generando un rompimiento de emulsión a bajas temperaturas y tiempo cortos, condiciones que no son aceptadas para la elaboración de emulsiones, se ensayaron 4 nuevas formulaciones con este aceite variando la cantidad de aceite y estabilizante, cada una con tres réplicas, sin embargo en el resultado no hubo variación, nuevamente, hubo rotura de la emulsión durante el proceso de emulsificación, que se pudo generar debido a la elevada concentración de ácidos grasos insaturados que tiene en su composición el aceite de Canola, considerando estos resultados se tomó la decisión de descartar el aceite de Canola para la elaboración del salchichón. 
Proceso de escaldado: Se realizó una nueva formulación con soya y girasol al $15 \%$ de composición en aceite para medir la temperatura máxima de estabilidad de la emulsión en escaldado, este proceso se realizó por triplicado y se sometió a las formulaciones a un rango de temperatura de $75^{\circ} \mathrm{C}-80^{\circ} \mathrm{C}$, hasta alcanzar una temperatura interna de $72^{\circ} \mathrm{C}$ en el producto (Ramírez, 2006; Lawrie, 2006), embutido en tripas de fibrosa de un diámetro de $6,5 \mathrm{~cm}$ y con un largo de $30 \mathrm{~cm}$, para un peso de $450 \mathrm{~g}$ en promedio. Realizado el procedimiento se encontró que no hubo ninguna alteración en los productos tales como: sinéresis, rotura de la emulsión, textura arenosa, etc. lo cual indicaba que soportaba las temperaturas establecidas en la teoría para la elaboración de productos escaldados y no necesitaba otras variaciones para alcanzar la cocción adecuada en el proceso.

Estandarización del producto: Luego de realizados los ensayos preliminares se pudieron estandarizar las tres formulaciones para el producto (Tabla 7). Se preparó un producto patrón, elaborado a partir de carne de res y grasa dorsal.

Tabla 7: Formulaciones finales para salchichón (g/100g)

\begin{tabular}{lrrrrrrrrr}
\hline Ingrediente & \multicolumn{3}{c}{ Formulación al 5\% } & \multicolumn{3}{c}{ Formulación al 10\% } & \multicolumn{3}{c}{ Formulación al 15\% } \\
\hline Aceite & 5 & 5 & 5 & 10 & 10 & 10 & 15 & 15 & 15 \\
Carne & 74.7 & 74.2 & 73.7 & 69.7 & 69.2 & 68.7 & 64.7 & 64.2 & 63.7 \\
Hielo & 12 & 12 & 12 & 12 & 12 & 12 & 12 & 12 & 12 \\
Harina & 4 & 4 & 4 & 4 & 4 & 4 & 4 & 4 & 4 \\
Proteína de soya & 1 & 1 & 1 & 1 & 1 & 1 & 1 & 1 & 1 \\
Sal & 1,5 & 1,5 & 1,5 & 1,5 & 1,5 & 1,5 & 1,5 & 1,5 & 1,5 \\
Sabor & 1,2 & 1,2 & 1,2 & 1,2 & 1,2 & 1,2 & 1,2 & 1,2 & 1,2 \\
Ajo en polvo & 0.05 & 0.05 & 0.05 & 0.05 & 0.05 & 0.05 & 0.05 & 0.05 & 0.05 \\
Humo & 0.05 & 0.05 & 0.05 & 0.05 & 0.05 & 0.05 & 0.05 & 0.05 & 0.05 \\
Estabilizante & 0.5 & 1 & 1.5 & 0.5 & 1 & 1.5 & 0.5 & 1 & 1.5 \\
\hline
\end{tabular}

Luego de elaboradas todas las muestras y puestas en almacenamiento a $4{ }^{\circ} \mathrm{C}$ los productos elaborados con el CMC al $1 \%$ y $1.5 \%$ estaban presentando una textura muy elástica, que los hacia diferenciar de los preparados al $0.5 \%$ en CMC, por esta razón se tomó la decisión de comprobar esta característica realizando una prueba de cizalla a los productos utilizando el texturómetro. Luego de realizada la prueba se obtuvieron los siguientes los resultados descritos en la Tabla 8.

Tabla 8: Resultados de textura en las primeras muestras de salchichón

\begin{tabular}{|l|l|l|l|l|l|l|l|l|}
\hline \multicolumn{3}{|c|}{ soya 5\% } & \multicolumn{3}{c|}{ soya 10\% } & \multicolumn{3}{c|}{ soya 15\% } \\
\hline \multicolumn{3}{|c|}{0 \% $\%$ est } & \multicolumn{3}{c|}{$1 \% \%$ est } \\
\hline rep1 & rep2 & rep3 & rep1 & rep2 & rep3 & rep1 & rep2 & rep3 \\
\hline $21.89 \mathrm{~N}$ & $22.35 \mathrm{~N}$ & $23.02 \mathrm{~N}$ & $32.55 \mathrm{~N}$ & $33.18 \mathrm{~N}$ & $32.89 \mathrm{~N}$ & $38.77 \mathrm{~N}$ & $38.92 \mathrm{~N}$ & $37.09 \mathrm{~N}$ \\
\hline \multicolumn{3}{|c|}{ girasol 5\% } & \multicolumn{3}{c|}{ girasol 10\% } & \multicolumn{3}{c|}{ girasol 15\% } \\
\hline \multicolumn{3}{|c|}{$0,5 \%$ est } & \multicolumn{3}{c|}{$1 \%$ est } & \multicolumn{3}{c|}{$1,5 \%$ est } \\
\hline rep1 & rep2 & rep3 & rep1 & rep2 & rep3 & rep1 & rep2 & rep3 \\
\hline $22.03 \mathrm{~N}$ & $23.26 \mathrm{~N}$ & $23.01 \mathrm{~N}$ & $34.97 \mathrm{~N}$ & $34.25 \mathrm{~N}$ & $34.91 \mathrm{~N}$ & $39.80 \mathrm{~N}$ & $40.06 \mathrm{~N}$ & $40.15 \mathrm{~N}$ \\
\hline
\end{tabular}

También se aplicó un análisis de textura a la muestra patrón para realizar un comparativo con los datos obtenidos y determinar si hay una diferencia significativa entre los productos preparados la Tabla 9 muestra resultados obtenidos de este análisis.

Tabla 9: Resultados de textura en las muestras de salchichón

\begin{tabular}{|c|l|l|l|l|l|l|l|l|}
\hline \multicolumn{3}{|c|}{ girasol 5\% } & \multicolumn{3}{c|}{ girasol 10\% } & \multicolumn{3}{c|}{ girasol 15\% } \\
\hline \multicolumn{3}{|c|}{$0,5 \%$ est } & \multicolumn{3}{c|}{$0,5 \%$ est est } \\
\hline rep1 & rep2 & rep3 & rep1 & rep2 & rep3 & rep1 & rep2 & rep3 \\
\hline $20.35 \mathrm{~N}$ & $19.85 \mathrm{~N}$ & $20.89 \mathrm{~N}$ & $18.88 \mathrm{~N}$ & $18.23 \mathrm{~N}$ & $18.45 \mathrm{~N}$ & $18.01 \mathrm{~N}$ & $17.95 \mathrm{~N}$ & $17.32 \mathrm{~B} \mathrm{~N}$ \\
\hline \multicolumn{3}{|c|}{ soya 5\% } & \multicolumn{3}{c|}{ soya 10\% } & \multicolumn{3}{c|}{ soya 15\% } \\
\hline \multicolumn{3}{|c|}{$0,5 \%$ est } & \multicolumn{3}{c|}{$1,0 \%$ est } & \multicolumn{3}{c|}{$1,5 \%$ est } \\
\hline rep1 & rep2 & rep3 & rep1 & rep2 & rep3 & rep1 & rep2 & rep3 \\
\hline $21.23 \mathrm{~N}$ & $20.95 \mathrm{~N}$ & $21.35 \mathrm{~N}$ & $20.32 \mathrm{~N}$ & $20.01 \mathrm{~N}$ & $20.89 \mathrm{~N}$ & $19.36 \mathrm{~N}$ & $20.35 \mathrm{~N}$ & $19.45 \mathrm{~N}$ \\
\hline
\end{tabular}


La variación más alta de textura utilizando el CMC se observa cuando del $5 \%$ se pasa al $10 \%$.De acuerdo con los resultados en el método ANOVA, ver tabla 10, se puede concluir que la textura cambia en la medida que se cambie el porcentaje de aceite tanto con el de soya como el de girasol, en las muestras con CMC.

Tabla 10: Análisis de Varianza Univariado (ANOVA) para evaluar el efecto sobre la textura del producto con los porcentajes de aceite de soya y girasol usados en su elaboración..

\begin{tabular}{|c|c|c|c|c|}
\hline Aceite & Valor de F & Grados & de libertad & $P$-valor \\
\hline adicionado & & Numerador & Denominador & \\
\hline Soya & 401,33 & 2 & 6 & $<, 0001$ \\
\hline Girasol & 1140,40 & 2 & 6 & $<, 0001$ \\
\hline
\end{tabular}

En la Tabla 11 se está mostrando una comparación de medias para cada porcentaje de aceite de soya y girasol y se encuentra que las medias por cada porcentaje son estadísticamente diferentes, pues cada una pertenece a un grupo diferente, siendo significativamente más altas en las muestras que incorporan grasa al $10 \%$ y $15 \%$, en presencia de CMC.

Tabla 11: Comparación de las medias obtenidas en cada nivel de grasa de soya y girasol usada en la elaboración del producto.

\begin{tabular}{cccc}
\hline Grupo & Porcentaje & \multicolumn{2}{c}{ Media } \\
\hline & & Soya & Girasol \\
A & $15 \%$ & 382.60 & 40.0 \\
B & $10 \%$ & 328.73 & 34.71 \\
C & $5 \%$ & 224.20 & 22.77 \\
\hline
\end{tabular}

Se encontró que los resultados de en textura son signifitativamente diferentes que los elaborados con el estabilizante al $1 \%$ y $1.5 \%$ y son similares a los del $0.5 \%$ de $\mathrm{CMC}$ en su formulacion. Esto se debe a que el CMC es un aditivo que a temperaturas mayores a $65^{\circ} \mathrm{C}$ tiende a gelificarse y esto afecta la estabilidad de la emulsion, por ende dando una textura mas elástica, la cual necesita una fuerza mayor de cizallamiento para romper su estructura, razón por la cual los datos de textura son mayores a los presentados en formulaciones al $0.5 \%$ de CMC y el producto patron, el cual no lleva este aditivo. Pero eliminando este aditivo de la formulación genera el rompimiento de la emulsión en escaldado, tal como se demostró elaborando un producto utilizando la formulación planteada pero sin este estabilizante. Por esta razon se tomó la determinacion de trabajar solo con la concentracion de CMC al $0,5 \%$.

Caracterización de los productos: A los productos terminados se les realizaron los análisis físicoquímicos en humedad, cenizas, proteína y grasa que se presentan en la Tabla 12. Las muestras se codificaron tomando la primera letra del nombre del aceite seguida por dos números que indican la composición porcentual del aceite en la formulación.

Tabla 12: Resultados de los análisis físico-químicos realizados a los productos terminados $(\mathrm{g} / 100 \mathrm{~g})$

\begin{tabular}{|c|c|c|c|c|}
\hline MUESTRA & HUMEDAD & CENIZAS & PROTEINA & GRASA \\
\hline G05 & 65.77 & 3.89 & 21.35 & 8.15 \\
\hline G10 & 62.48 & 3.20 & 18.95 & 13.58 \\
\hline G15 & 69.28 & 2.98 & 20.33 & 16.98 \\
\hline S05 & 60.53 & 3.02 & 18.05 & 8.17 \\
\hline S10 & 66.14 & 3.19 & 18.37 & 14.22 \\
\hline S15 & 64.47 & 3.15 & 18.81 & 16.84 \\
\hline
\end{tabular}

El resultado de la grasa aumenta a medida que sube el porcentaje de aceite presente en la muestra, lo que incidio en los resultados de proteína. Interpretando resultados de grasas y de textura se puede observar que a medida que el porcentaje de aceite aumenta en el producto 
disminuye la dureza, esta es una relación real ya que si aumenta el aceite, disminuye la cantidad de carne en el producto así como la fibra muscular y por consiguiente la dureza y la emulsión tiende a ser más suave.

Comparación de textura con dos productos del mercado: Se analizaron los datos de dureza de los salchichones, también por triplicado y se obtuvieron los resultados consignados en la Tabla 13

Tabla 13: Datos de dureza en las muestras de salchichón del mercado

\begin{tabular}{cc}
\hline MUESTRA & DUREZA (N) \\
\hline Sm1a-01 & 28.04 \\
Sm2a-02 & 27.95 \\
Sm3a-03 & 28.96 \\
Sm1b-01 & 38.95 \\
Sm2b-02 & 40.02 \\
Sm3b-03 & 39.16 \\
\hline
\end{tabular}

Las muestras de la investigación demostraron tener un nivel de dureza más bajo, lo que no sucede con los productos del mercado que contienen un contenido alto de extendedores en su formulación, estos aditivos tienen como objetivo aumentar el volumen del producto y de igual modo aumentan la elasticidad en el mismo y por ende una textura que necesita más fuerza de cizalla.

Análisis sensorial: Se aplicó una prueba hedónica con un panel no entrenado con 6 muestras evaluando color, aroma, sabor, apariencia y textura. Los resultados se presentan en la Figura 3

\section{Calificacion promedio de las muestras}

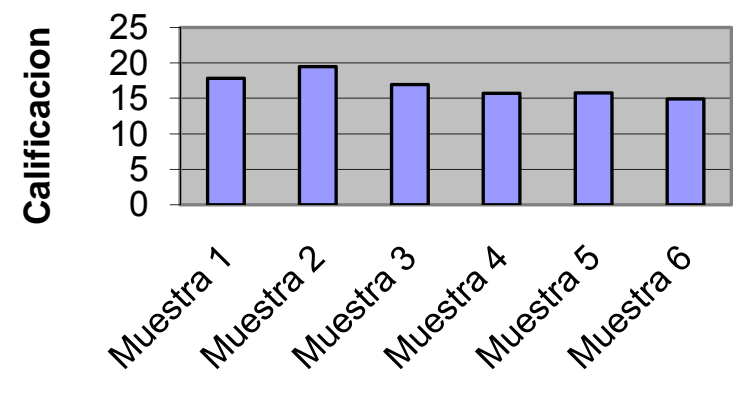

Fig. 3: Calificación promedio de las muestras de salchichón, en el análisis sensorial. Los autores.

La muestra 1 (S05) presentó una calificación promedio de 17.85, la muestra 2 (S10) de 19.5, la muestra 3 (S15) un valor de 16.95, la muestra 4 (G05) un total de 15.75 la muestra 5 (G10) da 15.8 y la muestra 6 (G15) un total de 14.9. Esto demuestra que la muestra que presenta la mejor combinación de características para los panelistas es la muestra 2 correspondiente al aceite de soya al $10 \%$. Las demás muestras estuvieron por debajo de la muestra seleccionada.

Perfil lipídico: La grasa es uno de los componentes mayoritarios en la elaboración de derivados cárnicos, la naturaleza de los ácidos grasos constitutivos de la grasa incide directamente sobre las características de calidad del producto, el conocer los ácidos grasos de los productos es fuente fundamental para corroborar la hipótesis planteada en la investigación. El método utilizado para la determinación de ácidos grasos se describe a continuación:

El perfil lipídico de la muestra de salchichón seleccionada (10\% aceite de soya), se realizó en el Laboratorio de Cromatografía de la Universidad Industrial de Santander, con el número de identificación 982253-01-AH, cuyos resultado se describen en la tabla numero 14. 
Tabla 14: Concentración de ácidos grasos, en la muestra de salchichón con 10\% aceite de soya

\begin{tabular}{l|c}
\hline Ácido graso & Concentración de ácidos graso (promedio) \\
\hline Caprílico (C8:0) & 1 \\
Cáprico & $<0.1$ \\
Láurico & 2 \\
Tridecanoico & $<0.1$ \\
Mirístico & 25 \\
Pentadecanoico & 11 \\
Palmítico & 1308 \\
Palmitoleico & 22 \\
Heptadecanoico & 41 \\
Esteárico & 731 \\
Elaídico & 5 \\
Oleíco (omega 9) & 2306 \\
Linolelaídico & 6 \\
Linoléico (omega 6) & 4929 \\
g - Linolénico & 1 \\
a- Linoléico (omega 3) & 558 \\
Araquídico & 41 \\
Eicosenoico & 22 \\
Eicosadienoico & 9 \\
Heneicosanoico & 4 \\
Eicosatrienoico & 10 \\
Araquidónico & 39 \\
Eicosapentaenoico & 14 \\
Behénico & 47 \\
Tricosanoico & 6 \\
\hline Lignocérico & 14 \\
\hline
\end{tabular}

Fuente: E. Stachenko, 2008 Lab. de cromatografía, UIS, B/manga.

En conclusión, los ácidos grasos saturados alcanzaron una concentración de $2229 \mathrm{mg} / 100 \mathrm{~g}$, los insaturados, $7908 \mathrm{mg} / 100 \mathrm{~g}$ de muestra, las grasas trans, $11 \mathrm{mg} / 100 \mathrm{~g}$ de muestra, para un gran total de ácidos grasos presentes en $100 \mathrm{~g}$ de muestra de salchichón al $10 \%$ de soya de $10148 \mathrm{mg}$.

Comparativo con productos del mercado: Los datos recogidos en la investigación seleccionaron al salchichón cuya composición tiene $10 \%$ en aceite de soya, la tabla 15 describe la composición fisicoquímica y de textura de estos dos derivados en comparativo con los del mercado.

Tabla 15: Comparativo del producto seleccionado, con dos salchichones del mercado.

\begin{tabular}{lccc}
\hline Indicador & Producto & Salchichón (S) & Salchichón (SB) \\
\hline Humedad (\%) & $64.60 \pm 2.3$ & $62.28 \pm 1.2$ & $61.23 \pm 2.3$ \\
Grasa (\%) & $13.63 \pm 1.0$ & $16.58 \pm 1.8$ & $18.86 \pm 0.9$ \\
$-\quad$ Saturada & $3.5 \%$ & 12.43 & 14.15 \\
- Insaturada & $10.13 \%$ & 4.15 & 4.71 \\
Proteína (\%) & $18.38 \pm 0.3$ & $14.54 \pm 1.8$ & $12.58 \pm 1.3$ \\
Dureza (N) & $22.81 \pm 4.2$ & $28.31 \pm 0.6$ & $39.37 \pm 0.6$ \\
\hline
\end{tabular}

Analizando la tabla anterior se pude observar como el producto seleccionado posee mayor cantidad de proteína y menor cantidad de grasa en su composición. Esta grasa del producto seleccionado está compuesta de ácidos grasos saturados en un $75 \%$, caracterizando el producto, no solo como bajo en grasa con respecto a los del mercado, sino que además con una gran mayoría compuesto de grasas saludables para la salud humana. 
En cuanto a los niveles de dureza se observa que el producto seleccionado es significativamente menor a los dos productos del mercado, resultado esperado ya que los productos del mercado tienen una cantidad más alta de aglutinantes (harinas y proteínas vegetales) que el producto seleccionado, estos componentes tienden a hacer el producto más elástico y por ende con una dureza mayor a la mordida.

Después de realizar el comparativo se puede determinar que la formulación seleccionada demuestra tener muchas ventajas frente a los productos del mercado, el cual puede convertirse en un producto cárnico funcional y saludable.

Análisis de vida útil: El análisis de vida útil se realizó por caracterización organoléptica, la cual consistió en tomar una muestra, observarla sensorialmente a los 5, 10, 15, 20, 25 y 30 días e ir tomando nota de los cambios a nivel del aspecto, aroma, textura y sabor. Estas muestras fueron siempre mantenidas a $4^{\circ} \mathrm{C}$ y con un envase protector transparente, que en el caso del salchichón es la fibrosa. A los 25 días presentó un olor bastante acentuado a rancio y en su sabor se percibía con claridad esta característica, razón fundamental para concluir que el tiempo de vida útil es de 25 días, el tiempo en el que el salchichón puede mantener sus características de calidad. Hay que tener en cuenta que para determinar la caducidad de los alimentos es necesario conocer los factores intrínsecos como materias primas, composición y formulación. Factores extrínsecos como control del almacenamiento, higiene y materiales de envasado y otros factores como manipulación.

\section{CONCLUSIONES}

Las carnes de búfalo y de res tienen características organolépticas muy similares pero la demanda en Colombia es muy restringida en el caso del búfalo a pesar de la bondad de esta carne en la salud del consumidor, ya que no existe la cultura de su consumo, al punto que algunos cortes de la canal es necesario pasarlos como carne de vacuno para su comercialización. Bos Indicus (2008). Esta última razón dio origen a la idea de procesar los cortes de segunda de la canal del búfalo y elaborar productos similares a los procesados cárnicos de la res pero con un valor agregado, el eliminado de la grasa libre saturada y la sustitución de ésta por grasas insaturadas que no solo texturizan el producto sino que lo enriquecen en ácidos grasos insaturados Omega 3, 6 y 9 que representan el $78 \%$ del total de grasa en el producto. El estudio se basó fundamentalmente en este objetivo y se elaboraron dos productos embutidos uno escaldado, el salchichón que hace parte de esta discusión.

El análisis fisicoquímico de la carne de búfalo demostró, que ésta posee niveles de proteína más altos que la carne de vacuno y a su vez unos niveles de grasa más bajos, lo cual indica que es una carne que tiene las condiciones para ser aprovechada industrialmente, su capacidad emulsionante CE es de $1115 \mathrm{~g}$ de aceite/g de proteína frente a la de vacuno con $1089 \mathrm{~g}$ de aceite/g de proteína en cuanto a la capacidad de retención de agua CRA posee las mimas características de pérdida de agua por goteo y por cocción que la carne de vacuno. La medida de dureza en la carne de búfalo es mayor que la de la carne de vacuno, característica coherente ya que la carne de búfalo posee una mayor cantidad de fibra muscular y menor cantidad de grasa razón que la hace más dura que una carne de diferente origen.

Los ensayos preliminares demostraron que los aceites utilizados en la investigación (soya y girasol) soportan las temperaturas de emulsificación y escaldado para la elaboración de derivados cárnicos escaldados, descritos por Lawrie (2006), lo mismo sucede con los tiempos de emulsificación propuestos ya que tampoco hay variación en el proceso, el aceite de canola no demostró poseer características funcionales para ser aprovechado en los procesos de elaboración del salchichón debido a su cantidad elevada de ácidos grasos insaturados, los cuales no son estables a efectos mecánicos y de cocción.

La muestra con formulación al $10 \%$ de soya, demostró que no solo es la que se destaca a nivel sensorial, si no que a su vez fue la que mantuvo los mejores niveles de terneza. Todas las muestras demostraron tener unos niveles de cenizas altos, por ende un alto nivel de minerales, 
dentro de los cuales se destaca el hierro ya que la carne de búfalo posee en su composición hasta un $12 \%$ más de hierro que la carne de los vacunos.

\section{AGRADECIMIENTOS}

Los autores agradecen a la Universidad De La Salle por la aprobación del presente proyecto de investigación, en cabeza de la Vicerrectora de Investigación y Transferencia. A la estadista Luz Marina Rondon por su aporte al desarrollo de esta investigación. Al programa de Ingeniería de Alimentos, Doctor Camilo Rozo, Decano de la misma, por su incondicional apoyo.

\section{REFERENCIAS}

Antunes, LC, Palheta A, Lourenço-Junior J, Felipe M. [CD-Room] Microbiological and sensorial characteristics of mortadela elaborated with "baby buffalo" meat and fine herbs. In: proceedings of the $1^{\text {st }}$ buffalo symposium of Americas Belém, Pará, Brazil. (2002).

Cedrés, J.F., Crudeli G.A., Patiño E.M., Rebak G.I., Bernardi A., Rivas P.A., Barrientos G.J. Composición química y características físicas de la carne de búfalos criados en forma extensiva en la provincia de Formosa. Disponible en: http://www.unne.edu.ar/cyt/2002/04-Veterinarias/V-040.pdf. (2002).

CODEX. Norma del CODEX para la carne picada curada cocida. CODEX STAN 98-1981 (Rev. 1. 1991) 6 pp. Disponible en: http://www.codexalimentarius.net/download/standards/161/CXS_098s.pdf. (1991).

Duxbury, D., Omega-3s Offer Solutions to Trans Fat Substitution problems. Food Technology. 59 (4): 34-39. (2005).

Escalante, J. La carne de búfalo una alternativa para una buena mesa y una buena alimentación. (http://salud.coomevacom.co/publicaciones. php?id=8130) (2008).

Guerrero, I., Tecnología de carnes. Ed trillas. México, D.F. p 25-29. (1998).

Hurtado-Lugo N., Restrepo L.F., Urrea A.D., Arcila M.P., Análisis sensorial de la carne bufalina (Bubalus bubalis) en la ciudad de Palmira, Colombia. Revista de Salud Pública y Nutrición 5(4) Disponible en: www.respyn.uanl.mx/v/4/articulos/analsis_sensorial_colombia_2004_1.htm. (2004).

ICONTEC. Industrias Alimentarias. Productos cárnicos procesados (no enlatados). Norma Técnica Colombiana NTC-1325. (1982).

Lawrie, R.A., Meat Science. Editorial Acribia. p 50-113. (2006).

Lees, R., Análisis de los Alimentos Métodos Analíticos y de Control de Calidad. Ed. Acribia S.A. Zaragoza, España. p 21-27; 61-250. (1982).

Lourenço J, Lourenço V, Costa N, Moura L, Lourenço LSousa, C, Santos N. [CD-Room] Evaluation of carcass income and physical-chemical Characteristics of the "baby buffalo" meat. In: proceedings of the $1^{\text {st }}$ buffalo symposium of Americas Belém, Brazil. (2002).

Ministerio de Salud. Decreto 2162, por el cual se reglamenta parcialmente el título $V$ de la ley 09 de 1979 , en cuanto a producción, procesamiento, transpor1e y expendio de los productos cárnicos procesados. Disponible en http://www.invima.gov.co/Invima///normatividad/docs_alimentos/decreto_2162_1983.pdf. (1983).

Ranken, M.D. Manual de Industrias de la Carne. Coedición AMV, Ediciones Mundiprensa 201 p. (2003).

Ramirez, R. Tecnología de carnicos. Ed. Unad. Bogota, Colombia. p 158-201. (2006).

Serrano A, Cofrades S., Productos Cárnicos más Saludables en el Ámbito Actual de los Alimentos funcionales. Alimentación Equipos y Tecnología. 200 (24): 50-54. (2005). 
